\title{
A Schmid-Leiman-Based Transformation Resulting in Perfect Inter-correlations of Three Types of Factor Score Predictors
}

André Beauducel

Institute of Psychology, University of Bonn, Germany, beauducel@uni-bonn.de

Follow this and additional works at: http://digitalcommons.wayne.edu/jmasm

Part of the Applied Statistics Commons, Social and Behavioral Sciences Commons, and the Statistical Theory Commons

\section{Recommended Citation}

Beauducel, A. (2017). A Schmid-Leiman-based transformation resulting in perfect inter-correlations of three types of factor score predictors. Journal of Modern Applied Statistical Methods, 16(1), 107-126. doi: 10.22237/jmasm/1493597160

This Regular Article is brought to you for free and open access by the Open Access Journals at DigitalCommons@WayneState. It has been accepted for inclusion in Journal of Modern Applied Statistical Methods by an authorized editor of DigitalCommons@WayneState. 


\section{A Schmid-Leiman-Based Transformation Resulting in Perfect Inter- correlations of Three Types of Factor Score Predictors}

\section{Erratum}

In the initial publication of this paper, the editors omitted the final term, $\operatorname{diag}\left(\Lambda^{\prime} \Sigma^{-1} \Lambda\right)^{1 / 2}$, from the first line of Equation 15 on page 114, placing it in the second line erroneously. This inversion has been corrected. 


\title{
A Schmid-Leiman-Based Transformation Resulting in Perfect Inter-correlations of Three Types of Factor Score Predictors
}

\author{
André Beauducel \\ University of Bonn \\ Bonn, Germany
}

Factor score predictors are computed when individual factor scores are of interest. Conditions for a perfect inter-correlation of the best linear factor score predictor, the best linear conditionally unbiased predictor, and the determinant best linear correlationpreserving predictor are presented. A transformation resulting in perfect correlations of the three predictors is proposed.

Keywords: $\quad$ Factor analysis, factor score predictors, Schmid-Leiman transformation

\section{Introduction}

Because factor scores are not determinate (Guttman, 1955), they cannot be unambiguously computed. However, factor score predictors can be computed as linear combinations of the observed variables in order to represent the individual scores of a latent variable. This might be useful when decisions have to be justified on the individual score level. Several different factor score predictors have meanwhile been proposed (Mulaik, 2010). The properties of different factor score predictors have been investigated by means of simulation studies (Fava \& Velicer, 1992) and by means of algebraic considerations (e.g. Beauducel \& Hilger, 2015; Krijnen, 2006; Krijnen, Wansbeek \& Ten Berge, 1996; McDonald \& Burr, 1967; Schneeweiss \& Mathes, 1995).

According to Grice (2001) and according to Krijnen et al. (1996) there are three main types of factor score predictors: The best linear predictor that is also known as Thurstone's (1935) regression predictor, the conditionally unbiased predictor (Krijnen et al., 1996; Bartlett, 1937), and the correlation-preserving predictor (McDonald, 1981; Ten Berge, Krijnen, Wansbeek, \& Shapiro, 1999).

André Beauducel is Faculty in the Institute of Psychology. Email at beauducel@unibonn.de . 


\section{PERFECT INTER-CORRELATIONS OF FACTOR SCORE PREDICTORS}

These three types of factor score predictors represent three desired properties: (a) The best linear predictor has a maximal correlation with the corresponding factor, (b) the conditionally unbiased predictor has zero correlations with noncorresponding factors, and (c) the correlation-preserving predictor has the advantage of preserving the correlations between the factors in the factor score predictor. The terms 'best linear predictor', 'conditionally unbiased predictor', and 'correlation-preserving predictor' are used as in Krijnen (2006).

McDonald and Burr (1967) explored the conditions for high correlations between factor score predictors for corresponding factors. They investigated the best linear predictor, a conditionally unbiased predictor, and a correlation preserving predictor. Since the determinant best linear correlation-preserving predictor (Ten Berge, Krijnen, Wansbeek, \& Shapiro, 1999) was not available at that time, they explored the Anderson-Rubin's (1956) orthogonal (orthogonality preserving) factor score predictor. They found that the three factor score predictors are perfectly correlated for the one-factor model (the Spearman case). The investigated factor score predictors are perfectly correlated in the case of unrotated canonical factor analysis (Rao, 1955). McDonald and Burr (1967) acknowledged the preference to use rotated factor loadings, because they can often be interpreted more easily. However, for the rotated factors the correlations between the factor score predictors would generally not be perfect, leading to the problem of choosing the optimal factor score predictor.

There are at least three types of factor score predictors corresponding to three different desired properties (Grice, 2001). Moreover, there are conditions for which the correlations between the factor score predictors are one for corresponding factors, so that no choice has to be made (McDonald \& Burr, 1967). It can be regarded as a substantial advantage of factor score predictors when they are simultaneously the best linear predictor, conditionally unbiased, as well as correlation preserving. Therefore, the aim of the present paper is (1) to explore further the conditions for perfect correlations between the factor score predictors of corresponding factors and (2) to propose a transformation method based on Schmid-Leiman (1957) that allows to find interpretable factors with perfect correlations between the three different types of factor score predictors.

\section{Methodology}

In order to present the equations defining the three factor score predictors, the definition of the population common factor model is given. The common factor 


\section{ANDRÉ BEAUDUCEL}

model assumes that $\mathbf{x}$, the random vector of observations of order $p$, is generated by

$$
\mathbf{x}=\boldsymbol{\Lambda} \mathbf{f}+\mathbf{e}
$$

where $\mathbf{f}$ is the random vector of factor scores of order $q$, $\mathbf{e}$ the random error vector of order $p$, and $\boldsymbol{\Lambda}$ the factor pattern matrix of order $p$ by $q$. The observations $\mathbf{x}$, the factor scores $\mathbf{f}$, and the error vectors $\mathbf{e}$ are assumed to have an expectation zero $(\varepsilon[\mathbf{x}]=0, \varepsilon[\mathbf{f}]=0, \varepsilon[\mathbf{e}]=0)$. The covariance between the factor scores and the error scores is assumed to be zero $(\operatorname{Cov}[\mathbf{f}, \mathbf{e}]=0)$. The standard deviation of $\mathbf{f}$ is one, the covariance of the observed variables is $\mathbf{x} \mathbf{x}^{\prime}=\mathbf{\Sigma}$. The covariance matrix $\boldsymbol{\Sigma}$ can be decomposed by

$$
\Sigma=\Lambda \Phi \Lambda^{\prime}+\Psi^{2}
$$

where $\boldsymbol{\Phi}$ represents the $q$ by $q$ factor correlation matrix and $\boldsymbol{\Psi}^{2}$ the $p$ by $p$ covariance matrix of the error scores $\mathbf{e}\left(\operatorname{Cov}[\mathbf{e}, \mathbf{e}]=\boldsymbol{\Psi}^{2}\right) . \Psi^{2}$ is assumed to be a diagonal matrix and it will be assumed in this paper that the matrix is positive definite.

The regression predictor or best linear (BL) predictor is given by $\hat{\mathbf{f}}_{\mathrm{BL}}=\boldsymbol{\Phi} \boldsymbol{\Lambda}^{\prime} \boldsymbol{\Sigma}^{-1} \mathbf{x}$. The condition $\mathbf{B}^{\prime} \boldsymbol{\Lambda}=\mathbf{I}$ holds for the class of conditionally unbiased predictors, where $\mathbf{B}$ are the weights for the factor score predictor (Bartlett, 1937). According to Krijnen et al. (1996), the best linear conditionally unbiased (BLCU) predictor is $\hat{\mathbf{f}}_{\mathrm{BCLU}}=\left(\boldsymbol{\Lambda}^{\prime} \boldsymbol{\Sigma}^{-1} \boldsymbol{\Lambda}\right)^{-1} \boldsymbol{\Lambda}^{\prime} \boldsymbol{\Sigma}^{-1} \mathbf{x}$. Ten Berge et al. (1999) defined a determinant best linear correlation-preserving (DBLCP) predictor, given by $\hat{\mathbf{f}}_{\mathrm{DBLCP}}=\boldsymbol{\Phi}^{1 / 2}\left(\boldsymbol{\Phi}^{1 / 2} \boldsymbol{\Lambda}^{\prime} \boldsymbol{\Sigma}^{-1} \boldsymbol{\Lambda} \boldsymbol{\Phi}^{1 / 2}\right)^{-1 / 2} \boldsymbol{\Phi}^{1 / 2} \Lambda^{\prime} \boldsymbol{\Sigma}^{-1} \mathbf{x}$. For this predictor symmetric positive (semi) definite matrices are raised to a certain power (e.g. square-root) by raising its eigenvalues to that power. When the power of the eigenvalues is $1 / 2$, this procedure is sometimes called the symmetric square-root (Harman, 1976).

\section{Results}

Conditions for a perfect correlation between $\hat{\mathbf{f}}_{\mathrm{BL}}, \hat{\mathbf{f}}_{\mathrm{BLCU}}$, and $\hat{\mathbf{f}}_{\mathrm{DBLCP}}$

The following Theorem 1 to 3 describe the conditions for perfect correlations between the factor score predictors for corresponding orthogonal factors. As will 


\section{PERFECT INTER-CORRELATIONS OF FACTOR SCORE PREDICTORS}

be shown in Theorem 4, a perfect correlation between the factor score predictors can only be found under unrealistic conditions when the factors are correlated. This is, of course, a limitation. However, the following Theorem 1 to 3 can nevertheless be applied to correlated factor solutions because correlated factor models can be transformed into corresponding orthogonal Schmid-Leiman (1957) models, as will be soon discussed.

Theorem 1 provides a condition for a perfect correlation between $\hat{\mathbf{f}}_{\mathrm{BCLU}}$ and $\hat{\mathbf{f}}_{\mathrm{BL}}$ for corresponding orthogonal factors.

Theorem 1. If $\boldsymbol{\Phi}=\mathbf{I}$ and $\Lambda^{\prime} \boldsymbol{\Sigma}^{-1} \boldsymbol{\Lambda}=\operatorname{diag}\left(\boldsymbol{\Lambda}^{\prime} \boldsymbol{\Sigma}^{-1} \boldsymbol{\Lambda}\right)$ then

$$
\varepsilon\left[\hat{\mathbf{f}}_{\mathrm{BCLU}} \hat{\mathbf{f}}_{\mathrm{BL}}^{\prime}\right] \operatorname{diag}\left(\varepsilon\left[\hat{\mathbf{f}}_{\mathrm{BCLU}} \hat{\mathbf{f}}_{\mathrm{BLCU}}^{\prime}\right]\right)^{-1 / 2} \operatorname{diag}\left(\varepsilon\left[\hat{\mathbf{f}}_{\mathrm{BL}} \hat{\mathbf{f}}_{\mathrm{BL}}^{\prime}\right]\right)^{-1 / 2}=\mathbf{R}_{\mathrm{BLCU}, \mathrm{BL}}=\mathbf{I} \text {. }
$$

Proof. The covariance between $\hat{\mathbf{f}}_{\mathrm{BCLU}}$ and $\hat{\mathbf{f}}_{\mathrm{BL}}$ is

$$
\mathrm{C}_{\mathrm{BLCU}, \mathrm{BL}}=\left(\Lambda^{\prime} \Sigma^{-1} \Lambda\right)^{-1} \Lambda^{\prime} \Sigma^{-1} \mathbf{x x}^{\prime} \Sigma^{-1} \Lambda \Phi=\Phi
$$

The correlation between $\hat{\mathbf{f}}_{\mathrm{BCLU}}$ and $\hat{\mathbf{f}}_{\mathrm{BL}}$ is therefore

$$
\mathbf{R}_{\mathrm{BLCU}, \mathrm{BL}}=\boldsymbol{\Phi} \operatorname{diag}\left(\left(\Lambda^{\prime} \Sigma^{-1} \Lambda\right)^{-1}\right)^{-1 / 2} \operatorname{diag}\left(\boldsymbol{\Phi} \Lambda^{\prime} \Sigma^{-1} \Lambda \Phi\right)^{-1 / 2}
$$

The element-wise square-root is calculated for the diagonal elements in Equation 4.

For $\boldsymbol{\Phi}=\mathbf{I}$ and $\boldsymbol{\Lambda}^{\prime} \boldsymbol{\Sigma}^{-1} \boldsymbol{\Lambda}=\operatorname{diag}\left(\boldsymbol{\Lambda}^{\prime} \boldsymbol{\Sigma}^{-1} \boldsymbol{\Lambda}\right)$, Equation 4 can be transformed into

$$
\mathbf{R}_{\mathrm{BLCU}, \mathrm{BL}}=\operatorname{diag}\left(\Lambda^{\prime} \Sigma^{-1} \Lambda\right)^{-1 / 2} \operatorname{diag}\left(\Lambda^{\prime} \Sigma^{-1} \Lambda\right)^{-1 / 2}=\mathbf{I} .
$$

This completes the proof.

The condition expressed in Theorem 1 is also a basis for a perfect correlation between $\hat{\mathbf{f}}_{\mathrm{DBLCP}}$ and $\hat{\mathbf{f}}_{\mathrm{BL}}$. 


\section{ANDRÉ BEAUDUCEL}

Theorem 2. If $\boldsymbol{\Phi}=\mathbf{I}$ and $\boldsymbol{\Lambda}^{\prime} \boldsymbol{\Sigma}^{-1} \boldsymbol{\Lambda}=\operatorname{diag}\left(\boldsymbol{\Lambda}^{\prime} \boldsymbol{\Sigma}^{-1} \boldsymbol{\Lambda}\right)$ then

$$
\varepsilon\left[\hat{\mathbf{f}}_{\mathrm{DBLCP}} \hat{\mathbf{f}}_{\mathrm{BL}}^{\prime}\right] \operatorname{diag}\left(\varepsilon\left[\hat{\mathbf{f}}_{\mathrm{DBLCP}} \hat{\mathbf{f}}_{\mathrm{DBLCP}}^{\prime}\right]\right)^{-1 / 2} \operatorname{diag}\left(\varepsilon\left[\hat{\mathbf{f}}_{\mathrm{BL}} \hat{\mathbf{f}}_{\mathrm{BL}}^{\prime}\right]\right)^{-1 / 2}=\mathbf{R}_{\mathrm{DBLCP}, \mathrm{BL}}=\mathbf{I} \text {. }
$$

Proof. The covariance between $\hat{\mathbf{f}}_{\mathrm{DBLCP}}$ and $\hat{\mathbf{f}}_{\mathrm{BL}}$ is

$$
\begin{aligned}
\mathrm{C}_{\mathrm{DBLCP}, \mathrm{BL}} & =\boldsymbol{\Phi}^{1 / 2}\left(\boldsymbol{\Phi}^{1 / 2} \Lambda^{\prime} \boldsymbol{\Sigma}^{-1} \Lambda \boldsymbol{\Phi}^{1 / 2}\right)^{-1 / 2} \boldsymbol{\Phi}^{1 / 2} \Lambda^{\prime} \boldsymbol{\Sigma}^{-1} \mathbf{X x}^{\prime} \boldsymbol{\Sigma}^{-1} \Lambda \boldsymbol{\Phi} \\
& =\boldsymbol{\Phi}^{1 / 2}\left(\boldsymbol{\Phi}^{1 / 2} \Lambda^{\prime} \boldsymbol{\Sigma}^{-1} \Lambda \boldsymbol{\Phi}^{1 / 2}\right)^{1 / 2} \boldsymbol{\Phi}^{1 / 2} .
\end{aligned}
$$

The corresponding correlation is

$$
\mathbf{R}_{\mathrm{DBLCP}, \mathrm{BL}}=\boldsymbol{\Phi}^{1 / 2}\left(\boldsymbol{\Phi}^{1 / 2} \Lambda^{\prime} \boldsymbol{\Sigma}^{-1} \Lambda \boldsymbol{\Phi}^{1 / 2}\right)^{1 / 2} \boldsymbol{\Phi}^{1 / 2} \operatorname{diag}\left(\boldsymbol{\Phi} \Lambda^{\prime} \boldsymbol{\Sigma}^{-1} \Lambda \Phi\right)^{-1 / 2} .
$$

For $\boldsymbol{\Phi}=\mathbf{I}$ and $\boldsymbol{\Lambda}^{\prime} \boldsymbol{\Sigma}^{-1} \boldsymbol{\Lambda}=\operatorname{diag}\left(\boldsymbol{\Lambda}^{\prime} \boldsymbol{\Sigma}^{-1} \boldsymbol{\Lambda}\right)$ Equation 7 can be transformed into

$$
\mathbf{R}_{\mathrm{DBLCP}, \mathrm{BL}}=\left(\Lambda^{\prime} \Sigma^{-1} \Lambda\right)^{1 / 2}\left(\Lambda^{\prime} \Sigma^{-1} \Lambda\right)^{-1 / 2}=\mathbf{I},
$$

because the symmetric square-root and the conventional square-root are identical for diagonal matrices. This completes the proof.

Finally, the condition presented in Theorem 1 and 2 is also the basis for a perfect correlation between $\hat{\mathbf{f}}_{\mathrm{BCLU}}$ and $\hat{\mathbf{f}}_{\mathrm{DBLCP}}$ for corresponding orthogonal factors.

Theorem 3. If $\boldsymbol{\Phi}=\mathbf{I}$ and $\boldsymbol{\Lambda}^{\prime} \boldsymbol{\Sigma}^{-1} \boldsymbol{\Lambda}=\operatorname{diag}\left(\boldsymbol{\Lambda}^{\prime} \boldsymbol{\Sigma}^{-1} \boldsymbol{\Lambda}\right)$ then $\varepsilon\left[\hat{\mathbf{f}}_{\mathrm{BCLU}} \hat{\mathbf{f}}_{\mathrm{DBLCP}}^{\prime}\right] \operatorname{diag}\left(\varepsilon\left[\hat{\mathbf{f}}_{\mathrm{BCLU}} \hat{\mathbf{f}}_{\mathrm{BLCU}}^{\prime}\right]\right)^{-1 / 2} \operatorname{diag}\left(\varepsilon\left[\hat{\mathbf{f}}_{\mathrm{DBLCP}} \hat{\mathbf{f}}_{\mathrm{DBLCP}}^{\prime}\right]\right)^{-1 / 2}=\mathbf{R}_{\mathrm{BLCU}, \mathrm{DBLCP}}=\mathbf{I}$.

Proof. The covariance between $\hat{\mathbf{f}}_{\mathrm{BCLU}}$ and $\hat{\mathbf{f}}_{\mathrm{DBLCP}}$ is

$$
\begin{aligned}
\mathrm{C}_{\mathrm{BLCU}, \mathrm{DBLCP}} & =\left(\Lambda^{\prime} \boldsymbol{\Sigma}^{-1} \Lambda\right)^{-1} \Lambda^{\prime} \boldsymbol{\Sigma}^{-1} \mathbf{x} \mathbf{x}^{\prime} \boldsymbol{\Sigma}^{-1} \Lambda \Phi^{1 / 2}\left(\Phi^{1 / 2} \Lambda^{\prime} \Sigma^{-1} \Lambda \Phi^{1 / 2}\right)^{-1 / 2} \boldsymbol{\Phi}^{1 / 2} \\
& =\Phi^{1 / 2}\left(\boldsymbol{\Phi}^{1 / 2} \Lambda^{\prime} \Sigma^{-1} \Lambda \Phi^{1 / 2}\right)^{-1 / 2} \Phi^{1 / 2} .
\end{aligned}
$$




\section{PERFECT INTER-CORRELATIONS OF FACTOR SCORE PREDICTORS}

The corresponding correlation is

$$
\mathbf{R}_{\mathrm{BLCU}, \mathrm{DBLCP}}=\boldsymbol{\Phi}^{1 / 2}\left(\boldsymbol{\Phi}^{1 / 2} \Lambda^{\prime} \boldsymbol{\Sigma}^{-1} \Lambda \boldsymbol{\Phi}^{1 / 2}\right)^{-1 / 2} \boldsymbol{\Phi}^{1 / 2} \operatorname{diag}\left(\left(\Lambda^{\prime} \boldsymbol{\Sigma}^{-1} \Lambda\right)^{-1}\right)^{-1 / 2} .
$$

If $\boldsymbol{\Phi}=\mathbf{I}$ and $\boldsymbol{\Lambda}^{\prime} \boldsymbol{\Sigma}^{-1} \boldsymbol{\Lambda}=\operatorname{diag}\left(\boldsymbol{\Lambda}^{\prime} \boldsymbol{\Sigma}^{-1} \boldsymbol{\Lambda}\right)$ Equation 10 can be transformed into

$$
\mathbf{R}_{\text {BLCU,DBLCP }}=\left(\Lambda^{\prime} \Sigma^{-1} \Lambda\right)^{-1 / 2}\left(\Lambda^{\prime} \Sigma^{-1} \Lambda\right)^{1 / 2}=\mathbf{I}
$$

This completes the proof.

Thus, the correlations between $\hat{\mathbf{f}}_{\mathrm{BL}}, \hat{\mathbf{f}}_{\mathrm{BCLU}}$ and $\hat{\mathbf{f}}_{\mathrm{DBLCP}}$, for corresponding orthogonal factors have been investigated for $\boldsymbol{\Phi}=\mathbf{I}$ and $\boldsymbol{\Lambda}^{\prime} \boldsymbol{\Sigma}^{-1} \boldsymbol{\Lambda}=\operatorname{diag}\left(\boldsymbol{\Lambda}^{\prime} \boldsymbol{\Sigma}^{-1} \boldsymbol{\Lambda}\right)$. It turned out $\hat{\mathbf{f}}_{\mathrm{BL}}, \hat{\mathbf{f}}_{\mathrm{BCLU}}$ and $\hat{\mathbf{f}}_{\mathrm{DBLCP}}$ are perfectly correlated for corresponding orthogonal factors with $\boldsymbol{\Lambda}^{\prime} \boldsymbol{\Sigma}^{-1} \boldsymbol{\Lambda}=\operatorname{diag}\left(\boldsymbol{\Lambda}^{\prime} \boldsymbol{\Sigma}^{-1} \boldsymbol{\Lambda}\right)$. Therefore, the interesting properties of these three types of factor score predictors can be obtained by a single set of factor score predictors under the conditions expressed in Theorems 1, 2 , and 3 .

Theorem 4 shows that it is possible to get a perfect correlation $\hat{\mathbf{f}}_{\mathrm{BL}}$ and $\hat{\mathbf{f}}_{\mathrm{BCLU}}$, for the correlated factors model, if at least some observed variables are measured without error.

Theorem 4. If $\boldsymbol{\Phi} \neq \operatorname{diag}(\boldsymbol{\Phi})$ then $\operatorname{diag}\left(\mathbf{R}_{\mathrm{BLCU}, \mathrm{BL}}\right)=\mathbf{I}$ if $\left(\boldsymbol{\Lambda}^{\boldsymbol{\top}} \boldsymbol{\Psi}^{-2} \boldsymbol{\Lambda}\right)^{-1}=\mathbf{0}$.

Proof. From Jöreskog (1969; Equation 10) we get $\boldsymbol{\Psi}^{-2} \boldsymbol{\Lambda}\left(\mathbf{I}+\boldsymbol{\Phi} \boldsymbol{\Lambda}^{\boldsymbol{}} \boldsymbol{\Psi}^{-2} \boldsymbol{\Lambda}\right)^{-1}=\boldsymbol{\Sigma}^{-1} \boldsymbol{\Lambda}$. Entering $\boldsymbol{\Psi}^{-2} \boldsymbol{\Lambda}\left(\mathbf{I}+\boldsymbol{\Phi} \boldsymbol{\Lambda}^{-} \boldsymbol{\Psi}^{-2} \boldsymbol{\Lambda}\right)^{-1}$ for $\boldsymbol{\Sigma}^{-1} \boldsymbol{\Lambda}$ into Equation 4 and some transformation yields

$$
\operatorname{diag}\left(\mathbf{R}_{\text {BLCU,BL }}\right)=\operatorname{diag}\left(\begin{array}{l}
\boldsymbol{\Phi} \operatorname{diag}\left(\left(\Lambda^{\prime} \Psi^{-2} \Lambda\right)^{-1}+\Phi\right)^{-1 / 2} \\
\operatorname{diag}\left(\Phi\left(\left(\Lambda^{\prime} \Psi^{-2} \Lambda\right)^{-1}+\Phi\right)^{-1} \Phi\right)^{-1 / 2}
\end{array}\right) .
$$




\section{ANDRÉ BEAUDUCEL}

For $\left(\boldsymbol{\Lambda}^{\prime} \boldsymbol{\Psi}^{-2} \boldsymbol{\Lambda}\right)^{-1}=\mathbf{0}$, Equation 12 yields

$$
\operatorname{diag}\left(\mathbf{R}_{\text {BLCU,BL }}\right)=\operatorname{diag}\left(\boldsymbol{\Phi} \operatorname{diag}(\boldsymbol{\Phi})^{-1 / 2} \operatorname{diag}(\boldsymbol{\Phi})^{-1 / 2}\right)=\mathbf{I} .
$$

This completes the proof.

The condition $\left(\boldsymbol{\Lambda}^{\prime} \boldsymbol{\Psi}^{-2} \boldsymbol{\Lambda}\right)^{-1}=\mathbf{0}$ can only be true if at least one observed variable of each factor is measured without error (Beauducel \& Hilger, 2015). This is, however, not realistic and it was therefore excluded in the definition of the factor model that $\boldsymbol{\Psi}$ contains zero elements. Although it cannot be excluded that some transformation methods might be found that allow to find correlated factor models with perfect correlations between $\hat{\mathbf{f}}_{\mathrm{BL}}$ and $\hat{\mathbf{f}}_{\mathrm{BCLU}}$, Theorem 4 demonstrates that this is impossible with conventional properties of $\left(\boldsymbol{\Lambda}^{\boldsymbol{}} \boldsymbol{\Psi}^{-2} \boldsymbol{\Lambda}\right)^{-1}$, which implies that the current approach is limited to orthogonal factor models. In order to overcome the limitation to orthogonal factor models Schmid-Leiman (1957) transformations of correlated factor models will be considered in the following.

\section{Transformation resulting in perfect correlations between $\hat{\mathbf{f}}_{\mathrm{BL}}, \hat{\mathrm{f}}_{\mathrm{BLCU}}$, and $\hat{\mathbf{f}}_{\mathrm{DBLCP}}$}

In the following, a transformation comprising four steps will be proposed that allows for orthogonal and correlated factors to be transformed into orthogonal (Schmid-Leiman) factors with perfect correlations between $\hat{\mathbf{f}}_{\mathrm{BL}}, \hat{\mathbf{f}}_{\mathrm{BCLU}}$ and $\hat{\mathbf{f}}_{\mathrm{DBLCP}}$. The transformation comprises four steps.

First, transform the factor loadings into

$$
\Lambda^{*}=\Lambda\left(\Lambda^{\prime} \Sigma^{-1} \Lambda\right)^{-1 / 2} \operatorname{diag}\left(\Lambda^{\prime} \Sigma^{-1} \Lambda\right)^{1 / 2}
$$

It follows that 


\section{PERFECT INTER-CORRELATIONS OF FACTOR SCORE PREDICTORS}

$$
\begin{aligned}
\Lambda^{* \prime} \Sigma^{-1} \Lambda^{*} & =\operatorname{diag}\left(\Lambda^{\prime} \Sigma^{-1} \Lambda\right)^{1 / 2}\left(\Lambda^{\prime} \Sigma^{-1} \Lambda\right)^{-1 / 2} \Lambda^{\prime} \Sigma^{-1} \Lambda\left(\Lambda^{\prime} \Sigma^{-1} \Lambda\right)^{-1 / 2} \operatorname{diag}\left(\Lambda^{\prime} \Sigma^{-1} \Lambda\right)^{1 / 2} \\
& =\operatorname{diag}\left(\Lambda^{\prime} \Sigma^{-1} \Lambda\right)
\end{aligned}
$$

which implies that $\boldsymbol{\Lambda}^{* \prime} \boldsymbol{\Sigma}^{-1} \boldsymbol{\Lambda}^{*}=\operatorname{diag}\left(\boldsymbol{\Lambda}^{* \prime} \boldsymbol{\Sigma}^{-1} \boldsymbol{\Lambda}^{*}\right)$ holds for $\boldsymbol{\Lambda}^{*}$.

Second, calculate the factor inter-correlations $\boldsymbol{\Phi}^{*}$ for the corresponding loadings, because the transformation by means of Equation 14 modifies the factor inter-correlations as long as $\boldsymbol{\Lambda}^{\prime} \boldsymbol{\Sigma}^{-1} \boldsymbol{\Lambda} \neq \mathbf{I}$, as follows from

$$
\begin{aligned}
\Phi & \neq \Phi^{*} \\
\left(\Lambda^{\prime} \Lambda\right)^{-1} \Lambda^{\prime}\left(\Sigma-\Psi^{2}\right) \Lambda\left(\Lambda^{\prime} \Lambda\right)^{-1} & \neq\left(\Lambda^{* \prime} \Lambda^{*}\right)^{-1} \Lambda^{* \prime}\left(\Sigma-\Psi^{2}\right) \Lambda^{*}\left(\Lambda^{* \prime} \Lambda^{*}\right)^{-1} \\
\left(\Lambda^{\prime} \Lambda\right)^{-1} \Lambda^{\prime}\left(\Sigma-\Psi^{2}\right) \Lambda\left(\Lambda^{\prime} \Lambda\right)^{-1} & \neq\left(\Lambda^{\prime} \Lambda\left(\Lambda^{\prime} \Sigma^{-1} \Lambda\right)^{-1 / 2} \operatorname{diag}\left(\Lambda^{\prime} \Sigma^{-1} \Lambda\right)^{1 / 2}\right)^{-1} \\
& \Lambda^{\prime}\left(\Sigma-\Psi^{2}\right) \Lambda \\
& \left(\operatorname{diag}\left(\Lambda^{\prime} \Sigma^{-1} \Lambda\right)^{1 / 2}\left(\Lambda^{\prime} \Sigma^{-1} \Lambda\right)^{-1 / 2} \Lambda^{\prime} \Lambda\right)^{-1}
\end{aligned}
$$

Thus, even when the initial factor model was orthogonal $(\boldsymbol{\Phi}=\mathbf{I})$, the transformed factor model will not necessarily be orthogonal $\left(\boldsymbol{\Phi}^{*} \neq \mathbf{I}\right)$. As already noted, the transformation of the loadings according to Equation 14 can also be performed for correlated factors. It is, however, possible that $\operatorname{diag}\left(\boldsymbol{\Phi}^{*}\right) \neq \mathbf{I}$ as should be because $\boldsymbol{\Phi}^{*}$ is a correlation matrix (see definition of the factor model). In order to make sure that $\operatorname{diag}\left(\boldsymbol{\Phi}^{*}\right)=\mathbf{I}$ it is necessary to rescale $\boldsymbol{\Lambda}^{*}$ by means of $\boldsymbol{\Lambda}^{*} \operatorname{diag}\left(\boldsymbol{\Phi}^{*}\right)^{-1 / 2}$ and to recalculate $\boldsymbol{\Phi}^{*}$ according to Equation 16 . According to Theorems 1 to 4 it is, moreover, necessary to have orthogonal factors in order to get perfect correlations between $\hat{\mathbf{f}}_{\mathrm{BL}}, \hat{\mathbf{f}}_{\mathrm{BCLU}}$ and $\hat{\mathbf{f}}_{\mathrm{DBLCP}}$ for corresponding factors.

Third, perform a second order factor analysis so that

$$
\Phi^{*}=\Lambda_{2}^{*} \Lambda_{2}^{* \prime}+\Psi_{2}^{* 2}
$$

where the subscript denotes the parameters of the second order factor model.

Fourth, perform a Schmid-Leiman (1957) transformation in order to compute orthogonal primary factors. It is possible to perform a Schmid-Leiman transformation of more complex hierarchical models. However, in purpose of 


\section{ANDRÉ BEAUDUCEL}

brevity it is assumed here that $\boldsymbol{\Phi}^{*}$ can be decomposed into a single general (second order) factor and the corresponding uniqueness of the primary factors, that is

$$
\boldsymbol{\Phi}^{*}=\Lambda_{2}^{*} \Lambda_{2}^{* \prime}+\Psi_{2}^{* 2}=\left[\begin{array}{lll}
\Lambda_{2}^{*} & \vdots & \Psi_{2}^{* 2}
\end{array}\right]\left[\begin{array}{c}
\Lambda_{2}^{*} \\
\Psi_{2}^{* 2}
\end{array}\right]=\mathbf{P P}^{\prime}
$$

The Schmid-Leiman transformation of the oblique first order factor model is

$$
\Lambda_{\mathrm{SL}}^{*}=\Lambda^{*} \mathbf{P}
$$

It follows from Equations 2, 18, and 19 that

$$
\Sigma=\Lambda^{*} \Phi^{*} \Lambda^{* \prime}+\Psi^{2}=\Lambda_{\mathrm{SL}}^{*} \Lambda_{\mathrm{SL}}^{*}+\Psi^{2}
$$

which implies that $\boldsymbol{\Lambda}_{\mathrm{SL}}$ represents the loadings of orthogonal factors. In the simplest Schmid-Leiman solution, the first column in $\boldsymbol{\Lambda}_{\mathrm{SL}}$ contains the loadings of the observed variables on a general (second order) factor that is orthogonal to the remaining orthogonalized primary factors.

However, the interest here is into the orthogonalized primary factors, which can be found in the columns 2 to $q$,

$$
\Lambda_{\mathbf{S L P}}^{*}=\left[\begin{array}{ccc}
\lambda_{\mathrm{SL}, 1,2}^{*} & \cdots & \lambda_{\mathrm{SL}, 1, q}^{*} \\
\vdots & \ddots & \vdots \\
\lambda_{\mathrm{SL}, p, 2}^{*} & \cdots & \lambda_{\mathbf{S L}, p, q}^{*}
\end{array}\right]
$$

The subset of orthogonalized primary factors can also be calculated by means of

$$
\Lambda_{\mathrm{SLP}}^{*}=\Psi_{2}^{2} \Lambda_{2}^{*}
$$

According to Equation 14 this implies

$$
\begin{aligned}
\Lambda_{\mathrm{SLP}}^{*}{ }^{\prime} \Sigma^{-1} \Lambda_{\mathrm{SLP}}^{*} & =\Psi_{2}^{* 2} \Lambda^{* \prime} \Sigma^{-1} \Lambda^{*} \Psi_{2}^{* 2} \\
& =\Psi_{2}^{* 2} \operatorname{diag}\left(\Lambda^{\prime} \Sigma^{-1} \Lambda\right) \Psi_{2}^{* 2}=\operatorname{diag}\left(\Lambda_{\mathbf{S L P}}^{*} \Sigma^{-1} \Lambda_{\mathbf{S L P}}^{*}\right)
\end{aligned}
$$




\section{PERFECT INTER-CORRELATIONS OF FACTOR SCORE PREDICTORS}

so that the conditions for perfect correlations of $\hat{\mathbf{f}}_{\mathrm{BL}}, \hat{\mathbf{f}}_{\mathrm{BCLU}}$ and $\hat{\mathbf{f}}_{\mathrm{DBLCP}}$ are met for the corresponding orthogonalized primary factors.

\section{Example}

A correlation matrix presented by Rimoldi (1948) based on 19 ability tests assessed in 138 participants was used in order to illustrate the transformation described above. As an initial factor model, principal axis factoring of the correlation matrix with subsequent oblique rotation (Promax, kappa =4) was performed with IBM SPSS Version 22 (see Table 1). The factor loading pattern and the factor inter-correlations were entered into the SPSS syntax presented in Appendix $\mathrm{A}$ in order to calculate the correlations between $\hat{\mathbf{f}}_{\mathrm{BL}}, \hat{\mathbf{f}}_{\mathrm{BCLU}}$ and $\hat{\mathbf{f}}_{\mathrm{DBLCP}}$ for the corresponding factors of the initial factor model. Appendix A also contains the four steps of the procedure described before and can be adapted for other data sets when the corresponding loading pattern and factor inter-correlations as well as the number of second order factors for the Schmid-Leiman solution is entered.

As can be seen from Table 2 the correlations between $\hat{\mathbf{f}}_{\mathrm{BL}}, \hat{\mathbf{f}}_{\mathrm{BCLU}}$ and $\hat{\mathbf{f}}_{\mathrm{DBLCP}}$ were already very high for the corresponding factors of the initial factor model. It should, however, be noted that the factor score predictors were based on exactly the same sample, the same observed variables and are thought to represent exactly the same factors. From this perspective especially some of the correlations between $\hat{\mathbf{f}}_{\mathrm{BL}}$ and $\hat{\mathbf{f}}_{\mathrm{BCLU}}$ indicate that the factor score predictors introduce a notable difference in the measurement of the same factors with the same participants. Therefore, a transformation of these factors according to the procedure described before was performed. 


\section{ANDRÉ BEAUDUCEL}

Table 1. Promax-rotated loading pattern and factor inter-correlations for 19 ability variables from Rimoldi (1948)

\begin{tabular}{|c|c|c|c|c|c|c|c|}
\hline Variable & F1 & F2 & F3 & F4 & F5 & F6 & F7 \\
\hline$\overline{1}$ & -.02 & .56 & -.08 & .01 & .00 & .12 & .12 \\
\hline 2 & -.11 & .38 & .33 & -.03 & .06 & .06 & -.04 \\
\hline 3 & .03 & .03 & -.03 & .67 & .00 & -.06 & -.22 \\
\hline 4 & -.04 & .18 & .54 & -.08 & .11 & -.25 & .24 \\
\hline 5 & -.02 & .35 & .01 & .20 & .13 & -.13 & .06 \\
\hline 6 & -.01 & .06 & .16 & .14 & .74 & -.07 & -.34 \\
\hline 7 & .04 & .02 & .02 & -.24 & .37 & .25 & .29 \\
\hline 8 & .10 & .15 & .25 & .25 & -.05 & -.11 & .24 \\
\hline 9 & .06 & .04 & .00 & -.09 & -.19 & -.06 & .47 \\
\hline 10 & -.01 & .18 & -.08 & .03 & -.02 & .67 & -.09 \\
\hline 11 & -.03 & -.13 & -.02 & .59 & .16 & .25 & .15 \\
\hline 12 & .06 & .45 & .07 & -.21 & .17 & .09 & -.19 \\
\hline 13 & -.01 & .37 & .29 & .03 & -.07 & .26 & -.10 \\
\hline 14 & .31 & -.10 & .65 & .01 & .14 & .02 & -.17 \\
\hline 15 & .65 & -.14 & .28 & .05 & -.09 & .10 & .00 \\
\hline 16 & .72 & -.12 & .15 & -.10 & .08 & -.07 & .15 \\
\hline 17 & .88 & .14 & .00 & .04 & -.12 & .02 & -.01 \\
\hline 18 & .59 & .17 & -.26 & .05 & .19 & -.05 & .01 \\
\hline 19 & .08 & .43 & .13 & .07 & -.18 & .05 & .16 \\
\hline \multicolumn{8}{|c|}{ factor inter-correlations } \\
\hline F2 & .37 & & & & & & \\
\hline F3 & .52 & .22 & & & & & \\
\hline F4 & .37 & .18 & .39 & & & & \\
\hline F5 & .28 & .17 & .25 & .19 & & & \\
\hline F6 & .02 & -.12 & .17 & .02 & .19 & & \\
\hline F7 & .29 & .04 & .35 & .35 & .52 & .45 & \\
\hline
\end{tabular}

Note. Loadings with an absolute size $\geq .30$ are given in bold face.

Table 2. Correlations between $\hat{\mathbf{f}}_{\mathrm{BL}}, \hat{\mathbf{f}}_{\mathrm{BLCU}}$, and $\hat{\mathbf{f}}_{\mathrm{DBLCP}}$ for the corresponding factors of the initial factor model

\begin{tabular}{lrrrrrrr} 
& F1 & F2 & F3 & F4 & F5 & F6 & F7 \\
\hline$\hat{\mathbf{f}}_{\mathrm{BL}}$ with $\hat{\mathbf{f}}_{\mathrm{BLCU}}$ & .993 & .986 & .968 & .983 & .979 & .984 & .947 \\
$\hat{\mathbf{f}}_{\mathrm{BL}}$ with $\hat{\mathbf{f}}_{\mathrm{DBLCP}}$ & .999 & .996 & .992 & .995 & .995 & .996 & .987 \\
$\hat{\mathbf{f}}_{\mathrm{BLCU}}$ with $\hat{\mathbf{f}}_{\mathrm{DBLCP}}$ & .998 & .997 & .992 & .996 & .995 & .996 & .986 \\
\hline
\end{tabular}

In the first step of the transformation described above, the factor loading pattern was transformed according to Equation 14 (see Appendix A). In the second step, the factor inter-correlations were calculated for the transformed loading pattern 


\section{PERFECT INTER-CORRELATIONS OF FACTOR SCORE PREDICTORS}

(Equation 16). The loading pattern and the factor inter-correlations were rescaled. Third, an unrotated second order principal axis factoring of the inter-correlations of the factors was performed. A single second order factor was extracted. Fourth, a Schmid-Leiman solution was computed from the second order factor and the transformed primary factors (Equation 19; see Table 3). It turned out that the loading pattern of the initial primary factors and the loading pattern of the transformed Schmid-Leiman primaries were similar, which implies that the interpretation of the factors was not substantially altered by the transformations. The correlations between $\hat{\mathbf{f}}_{\mathrm{BL}}, \hat{\mathbf{f}}_{\mathrm{BCLU}}$ and $\hat{\mathbf{f}}_{\mathrm{DBLCP}}$ for the corresponding primary factors presented in Table 3 were all perfect $(=1.000)$ so that an additional table was not necessary.

Table 3. Schmid-Leiman model of the primary factors transformed according to (14)

\begin{tabular}{|c|c|c|c|c|c|c|c|c|}
\hline \multirow[b]{2}{*}{ Variable } & \multirow{2}{*}{$\begin{array}{r}2^{\text {nd }} \\
\text { order } \\
\text { factor } \\
\mathrm{F} 1\end{array}$} & \multicolumn{7}{|c|}{ Primary Factors } \\
\hline & & F1 & F2 & F3 & F4 & F5 & F6 & F7 \\
\hline$\overline{1}$ & .15 & .05 & .52 & -.03 & .04 & .06 & .09 & .10 \\
\hline 2 & .16 & .01 & .37 & .29 & .02 & .06 & .05 & .00 \\
\hline 3 & .06 & .09 & .07 & .03 & .60 & -.02 & -.10 & -.12 \\
\hline 4 & .30 & .11 & .22 & .48 & .03 & .15 & -.17 & .21 \\
\hline 5 & .14 & .07 & .36 & .04 & .21 & .14 & -.14 & .07 \\
\hline 6 & .18 & .10 & .12 & .14 & .13 & .61 & -.08 & -.13 \\
\hline 7 & .33 & .08 & .01 & .05 & -.17 & .40 & .29 & .30 \\
\hline 8 & .30 & .20 & .19 & .28 & .31 & .03 & -.08 & .21 \\
\hline 9 & .16 & .07 & .03 & .03 & -.02 & -.08 & -.01 & .33 \\
\hline 10 & .15 & -.01 & .12 & -.02 & .00 & .00 & .59 & -.01 \\
\hline 11 & .36 & .05 & -.11 & .07 & .55 & .19 & .25 & .22 \\
\hline 12 & .03 & .10 & .43 & .06 & -.18 & .14 & .04 & -.13 \\
\hline 13 & .17 & .07 & .35 & .28 & .06 & -.05 & .22 & -.04 \\
\hline 14 & .31 & .39 & -.01 & .60 & .09 & .14 & .05 & -.04 \\
\hline 15 & .30 & .61 & -.06 & .34 & .13 & -.02 & .11 & .06 \\
\hline 16 & .33 & .67 & -.02 & .23 & .02 & .16 & -.03 & .17 \\
\hline 17 & .26 & .80 & .23 & .14 & .13 & -.02 & .00 & .02 \\
\hline 18 & .18 & .53 & .23 & -.13 & .09 & .23 & -.07 & .05 \\
\hline 19 & .19 & .15 & .42 & .17 & .12 & -.10 & .04 & .12 \\
\hline
\end{tabular}

Note. Loadings with an absolute size $\geq .30$ are given in bold face. 


\section{ANDRÉ BEAUDUCEL}

\section{Conclusion}

Conditions were explored for a perfect correlation between three types of factor score predictors: The regression predictor or best linear predictor, the conditionally unbiased best linear predictor, and the determinant best linear correlation-preserving predictor. A perfect correlation between these factor score predictors for corresponding factors implies that the choice between these factor score predictors does not matter and that each type of factor score predictor will have the virtues of the other. That is, the conditionally unbiased best linear predictor will also be the best linear predictor, the determinant best linear correlation-preserving predictor, will have the virtue to be conditionally unbiased predictor, etc. Thus, the conditions of a perfect correlation between the three types of factor score predictors for corresponding factors might be of interest for applied researchers, who want to calculate score predictors combining the different advantages.

McDonald and Burr (1967) found three types of factor score predictors similar to the predictors investigated here are perfectly correlated for one-factor models and for the unrotated canonical factor model. In addition to these conditions, it was shown here that for orthogonal factors with $\Lambda^{\prime} \boldsymbol{\Sigma}^{-1} \Lambda=\operatorname{diag}\left(\Lambda^{\prime} \boldsymbol{\Sigma}^{-1} \Lambda\right)$ the three factor score predictors are perfectly correlated. A method for transforming a loading matrix according to this condition was proposed. The transformation can also be applied to models with correlated factors. Moreover, the factors resulting from this transformation are not necessarily orthogonal. Since it has been shown that the factors corresponding to $\Lambda^{\prime} \boldsymbol{\Sigma}^{-1} \Lambda=\operatorname{diag}\left(\Lambda^{\prime} \boldsymbol{\Sigma}^{-1} \Lambda\right)$ should be orthogonal in order to provide perfect correlations between the three types of factor score predictors for corresponding factors a hierarchical Schmid-Leiman solution was computed. Thereby the correlated factor models are transformed into a combined solution of orthogonal second order factors and orthogonal primary factors. Since the Schmid-Leiman transformation can be applied to any hierarchical pattern of loading matrices, the transformation method proposed here can also be applied to confirmatory factor models.

The results of the current study show that it is possible to obtain a single set of factor score predictors that combine the virtues of the best linear predictor, of the conditionally unbiased predictor, and of the correlation-preserving predictor. This may be of interest for research and applications, where a high quality of the factors score predictors is of special importance. 


\section{PERFECT INTER-CORRELATIONS OF FACTOR SCORE PREDICTORS}

As an example, the transformation was applied to the data set of Rimoldi (1948), who published the correlation matrix of 19 ability measures. The

corresponding SPSS syntax (Appendix A) can be adapted in order to be used for other data sets.

\section{References}

Anderson, T. W. \& Rubin, H. (1956). Statistical inference in factor analysis. Proceedings of the Third Berkeley Symposium of Mathematical Statistics and Probability, 5, 111-150.

Bartlett, M. S. (1937). The statistical conception of mental factors. British Journal of Psychology, 28(1), 97-104. doi: 10.1111/j.2044-8295.1937.tb00863.x

Beauducel, A. \& Hilger, N. (2015). Extending the debate between Spearman and Wilson 1929: When do single variables optimally reproduce the common part of the observed covariances? Multivariate Behavioral Research, 50(5), 555-567. doi: 10.1080/00273171.2015.1059311

Fava, J. L. \& Velicer, W. F. (1992). An empirical comparison of factor, image, component, and scale scores. Multivariate Behavioral Research, 27(3), 301-322. doi: 10.1207/s15327906mbr2703_1

Guttman, L. (1955). The determinacy of factor score matrices with applications for five other problems of common factor theory. British Journal of Statistical Psychology, 8(2), 65-82. doi: 10.1111/j.2044-8317.1955.tb00321.x

Grice, J. W. (2001). Computing and evaluating factor scores. Psychological Methods, 6(4), 430-450. doi: 10.1037/1082-989X.6.4.430

Harman, H. H. (1976). Modern Factor Analysis (3rd ed.). Chicago: The University of Chicago Press.

Jöreskog, K. G. (1969). A general approach to confirmatory maximum likelihood factor analysis. Psychometrika, 34(2), 183-202. doi: 10.1007/bf02289343

Krijnen, W. P. (2006). Some results on mean square error for factor score prediction. Psychometrika, 71(2), 395-409. doi: 10.1007/S 11336-004-1220-7

Krijnen, W. P., Wansbeek, T.J., \& Ten Berge, J.M.F. (1996). Best linear predictors for factor scores. Communications in Statistics: Theory and Methods, 25(12), 3013-3025. doi: 10.1080/03610929608831883

McDonald, R. P. (1981). Constrained least squares estimators of oblique common factors. Psychometrika, 46(3), 337-341. doi: 10.1007/BF02293740 


\section{ANDRÉ BEAUDUCEL}

McDonald, R. P. \& Burr, E. J. (1967). A comparison of four methods of constructing factor scores. Psychometrika, 32(4), 381-401. doi:

10.1007/BF02289653

Mulaik, S. A. (2010). Foundations of factor analysis (2nd Ed.). New York: CRC Press.

Rao, C. R. (1955). Estimation and tests of significance in factor analysis. Psychometrika, 20(2), 93-112. doi: 10.1007/BF02288983

Rimoldi, H. J. (1948). Study of some factors related to intelligence. Psychometrika, 13(1), 27-46. doi: 10.1007/BF02288945

Schmid, J. \& Leiman, J. M. (1957). The development of hierarchical factor solutions. Psychometrika, 22(1), 53-61. doi: 10.1007/BF02289209

Schneeweiss, H. \& Mathes, H. (1995). Factor Analysis and Principal Components. Journal of Multivariate Analysis, 55(1), 105-124. doi: 10.1006/jmva.1995.1069

Ten Berge, J. M. F., Krijnen, W. P., Wansbeek, T., Shapiro, A. (1999). Some new results on correlation-preserving factor scores prediction methods. Linear Algebra and its Applications, 289(1-3), 311-318. doi: 10.1016/S00243795(97)10007-6

Thurstone, L.L. (1935). The Vectors of Mind. Chicago: University of Chicago Press. 


\section{PERFECT INTER-CORRELATIONS OF FACTOR SCORE PREDICTORS}

\section{Appendix A}

set MXLOOPS $=1000$ workspace $=400000$.

MATRIX.

* enter initial loading pattern into l:.

compute $L=\{$

$-0.019,0.555,-0.083,0.012,0.005,0.122,0.123$;

$-0.108,0.384,0.334,-0.028,0.064,0.060,-0.042$;

$0.033,0.027,-0.033,0.671,0.002,-0.059,-0.220$;

$-0.035,0.183,0.541,-0.079,0.105,-0.246,0.244$;

$-0.024,0.353,0.007,0.196,0.129,-0.140,0.063$;

$-0.014,0.059,0.164,0.141,0.744,-0.074,-0.343$;

$0.044,0.020,0.021,-0.236,0.371,0.251,0.291$;

$0.098,0.154,0.250,0.254,-0.046,-0.112,0.236$;

$0.064,0.039,-0.005,-0.086,-0.192,-0.065,0.470$;

$-0.008,0.176,-0.079,0.030,-0.022,0.667,-0.089$;

$-0.030,-0.128,-0.021,0.593,0.163,0.252,0.146$;

$0.058,0.447,0.067,-0.214,0.170,0.088,-0.190$;

$-0.012,0.366,0.290,0.027,-0.073,0.264,-0.100$;

$0.315,-0.095,0.647,0.007,0.146,0.024,-0.170$;

$0.648,-0.146,0.280,0.049,-0.087,0.101,0.001$;

$0.719,-0.121,0.152,-0.095,0.075,-0.074,0.153$;

$0.879,0.141,0.002,0.039,-0.117,0.016,-0.012$;

$0.586,0.170,-0.265,0.050,0.186,-0.053,0.011$;

$0.076,0.434,0.128,0.069,-0.176,0.050,0.164\}$.

* ENTER INITIAL FACTOR INTER-CORRELATIONS INTO PHI:. compute $\mathrm{Phi}=\{$

$1.000,0.366,0.518,0.372,0.279,0.017,0.285$;

$0.366,1.000,0.219,0.180,0.170,-0.118,0.035$;

$0.518,0.219,1.000,0.385,0.246,0.173,0.348$;

$0.372,0.180,0.385,1.000,0.192,0.019,0.353$;

$0.279,0.170,0.246,0.192,1.000,0.186,0.521$;

$0.017,-0.118,0.173,0.019,0.186,1.000,0.449$;

$0.285,0.035,0.348,0.353,0.521,0.449,1.000\}$. 


\section{ANDRÉ BEAUDUCEL}

* enter nUMBer OF SECOND ORDER FACTORS.

compute nF_2nd=1.

compute Psi2=Mdiag(diag( ident (nrow $(L)$, nrow(L)) - L*Phi*T(L) )).

compute Sig=L*Phi*T(L) + Psi2.

Print /Title "Initial factor loading pattern:".

print $\{\mathrm{L}\} /$ format=F5.2.

Print /Title "Initial factor inter-correlations:".

print $\{$ Phi $\} /$ format=F5.2.

Print /Title "Number of factors for second order factor analysis:".

print nf_2nd/format=F2. $\theta$.

Print /Title "Initial correlation between BLCU and BL factor score predictor (Equation 4):".

compute EQ4=Phi*INV(Mdiag(diag( $\operatorname{INV}(\mathrm{T}(\mathrm{L}) * \operatorname{INV}(\mathrm{Sig}) * \mathrm{~L})))) \& * *(0.5) *$

$\operatorname{INV}\left(\operatorname{Mdiag}\left(\operatorname{diag}\left(\mathrm{Phi}^{*} \mathrm{~T}(\mathrm{~L}) * \operatorname{INV}(\mathrm{Sig}) * \mathrm{~L} * \mathrm{Phi}\right)\right)\right) \& * *(0.5)$.

print EQ4/format=F6.3.

Print /Title "Initial correlation between DBLCP and BL factor score predictor (Equation 7):".

CALL SVD(Phi, q, eig, qq).

compute Phi12 $=q^{*}($ eig\&**0.5)*T $(q)$.

compute $\mathrm{H}=\mathrm{Phi12} * \mathrm{~T}(\mathrm{~L}) * \mathrm{INV}(\mathrm{Sig}) * \mathrm{~L} * \mathrm{Phi12}$.

$\operatorname{CALL~SVD}(H, q$, eig, qq).

compute $\mathrm{H} 12=\mathrm{q} *($ eig\&**0.5)*T(q).

compute EQ7=Phi12*H12*Phi12*INV(Mdiag(diag(Phi*T(L)*INV(Sig)*L*Phi)))\&**(0.5).

print EQ7/format=F6.3.

Print /Title "Initial correlation between BLCU and DBLCP factor score predictor (Equation 10):".

compute EQ10=Phi12*INV(H12)*Phi12*INV(Mdiag(diag(INV(T(L)*INV(Sig)*L))) )\&*(0.5). print EQ10/format=F6.3. 


\section{PERFECT INTER-CORRELATIONS OF FACTOR SCORE PREDICTORS}

* TRANSFORMATION OF PRIMARY FACTORS: .

* STEP 1 - Compute transformed loadings according to Equation 14. compute help $=\mathrm{T}(\mathrm{L}) * \operatorname{INV}(\mathrm{Sig}) * \mathrm{~L}$.

CALL SVD(help, V, Eig, TV).

compute help12=V*(Eig\&**0.5)*T(V).

compute $\mathrm{L} 14=\mathrm{L} \quad * \operatorname{INV}($ help12)*Mdiag(diag $(\mathrm{T}(\mathrm{L}) * \operatorname{INV}(\mathrm{Sig}) * \mathrm{~L})) \& * * 0.5$.

* STEP 2 - Compute factor intercorrelations and rescale transformed loadings. compute Phi14=INV(T(L14)*L14)*T(L14)*L*Phi*T(L) *L14*INV(T(L14)*L14).

compute L14=L14*(Mdiag(diag(Phi14)))\&**0.5.

Print /Title "STEP $1+2$ - Loading pattern of rescaled transformed primary

factors:".

Print L14/format=F5.2.

compute Phi14=INV(T(L14)*L14)*T(L14)*L*Phi*T(L) *L14*INV(T(L14)*L14).

Print /Title "STEP $1+2$ - Inter-correlations of transformed primary factors:".

Print Phi14/format=F5.2.

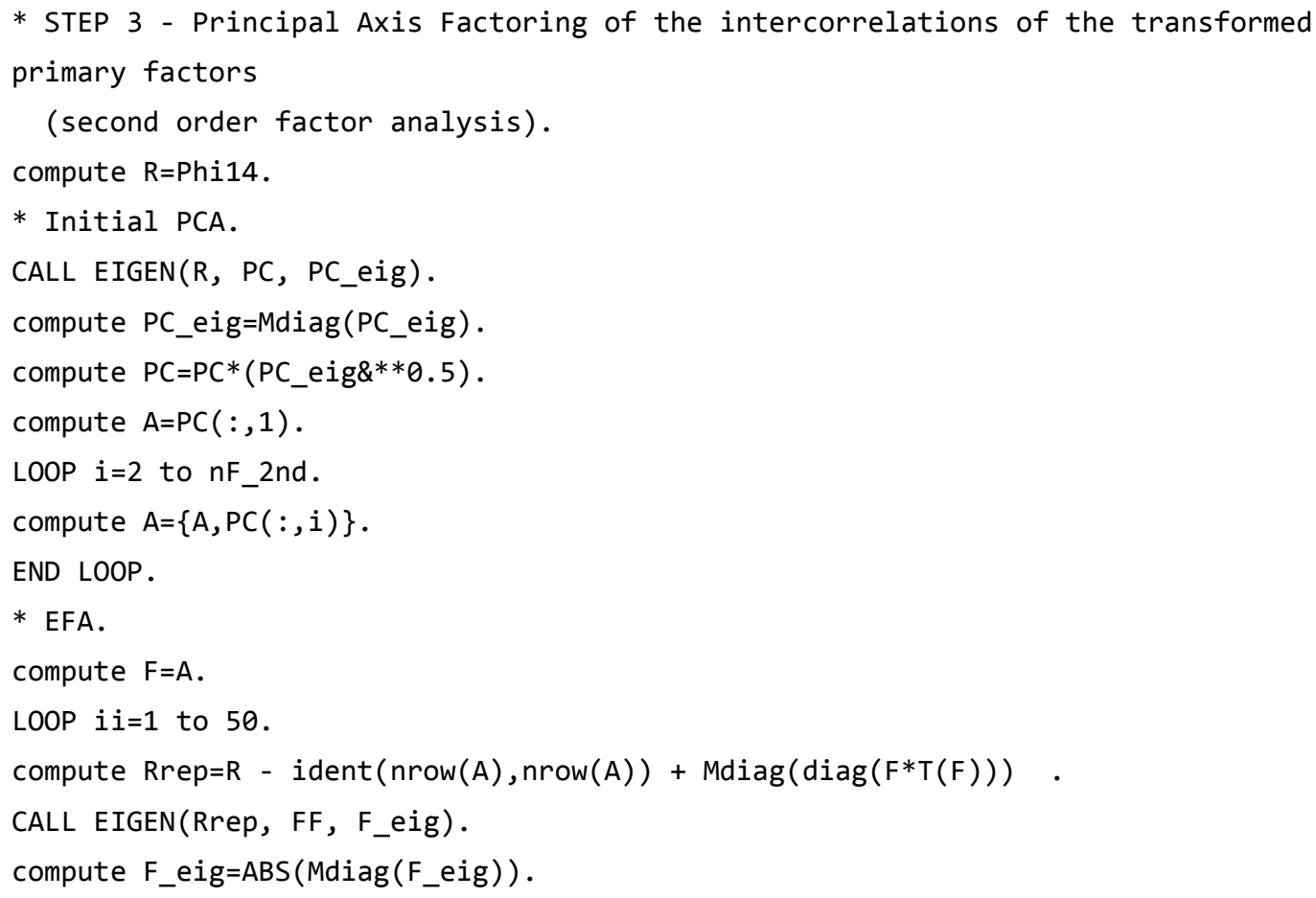




\section{ANDRÉ BEAUDUCEL}



* CHECK: Compute the inter-correlations between factor score predictors for the primaries.

* SELECT PRIMARIES OF SCHMID-LEIMAN SOLUTION: .

compute SL_p=SL14 $(:, 2)$.

LOOP $\mathrm{i}=\mathrm{nF} \_2 \mathrm{nd}+2$ to $\mathrm{nF} \_2 \mathrm{nd}+\mathrm{ncol}(\mathrm{L})$.

compute SL_p=\{SL_p, SL14 $(:, i)\}$.

END LOOP.

Print /Title "Correlation between BLCU and BL factor score predictor (Equation 4) for transformed primaries:". 


\section{PERFECT INTER-CORRELATIONS OF FACTOR SCORE PREDICTORS}

$\operatorname{CALL} \operatorname{SVD}(H, q$, eig, qq).

compute $\mathrm{H} 12=\mathrm{q} *($ eig\&**0.5)*T(q).

compute EQ7_14=H12*INV(Mdiag(diag(T(SL_p)*INV(Sig)*SL_p)))\&**(0.5).

print EQ7_14/format=F6.3.

Print /Title "Correlation between BLCU and DBLCP factor score predictor

(Equation 10) for transformed primaries:".

compute EQ10_14=INV(H12)*INV(Mdiag(diag(INV(T(SL_p)*INV(Sig)*SL_p))))\&**(0.5).

print EQ10_14/format=F6.3.

Print /Title

"Weights (B) for computation of factor scores as fscore=T(B)*Z, with Z "

+ "containing z-standardized variables with rows=variables, columns=cases". compute $B=\operatorname{INV}(\operatorname{Sig}) * S L \_p$.

print B/format=F6.3.

* For calculating the factor scores delete the first "*" in the three lines starting with "get".

* Enter the number of z-standardized variables in "\#\#" and the file handle in "...".

*get Z / variables= Z1 to Z\#\# /file="C:\..\zscores.sav".

* compute Fscores $=\mathrm{Z} * \mathrm{~B}$.

*save $\{$ Fscores $\}$ /outfile="C: \...\Fscores.sav".

END MATRIX. 